\title{
Efficiency of sorbents used to restore the grip of the surface of the oily road
}

\author{
Mirosław Sobolewski ${ }^{1}$, Dominika Gancarczyk ${ }^{1,{ }^{*}}$, and Piotr Książek ${ }^{1}$ \\ ${ }^{1}$ The Main School of Fire Service, Faculty of Fire Safety Engineering, 52/54 Slowackiego St., 01-629 \\ Warsaw, Poland
}

\begin{abstract}
The use of sorbents is one of the methods most commonly used by the fire departments to remove spilled hydrocarbon liquids. Sorbents approved for use by fire brigades must meet the requirements of the Ministry of Internal Affairs. However, this requirement does not include the assessment of the degree of roughness of the road surface after removal of oily spills. The high degree of restoration of road surface grip is extremely important for the safety of road users. The article presents the results of research on the effectiveness of restoring the coefficient of friction by different sorbents. The tests were carried out for three different asphalt pavements, determining the coefficients of friction for dry, wet and oily surfaces and after applying sorbents. Static friction coefficients were determined by measuring the angle of the inclination of the tested surface specimen, at which the probe imitating the car tire slid. It turned out that none of the used sorbents fully restored the initial coefficient of friction on the asphalt surface. The use of professional sorbents allowed for the restoration of about $80 \%$ of the initial coefficient of friction of the dry surface. Significantly poorer results were obtained for the most commonly used sorptive replacement materials, i.e. sand or sawdust.
\end{abstract}

\section{Introduction}

In recent years, much attention has been paid to the anti-slip properties of road surfaces, which have a direct impact on the safety of road users. Appropriate roughness of the surface is responsible for both the movement of vehicles and the length of the braking distance. Wet surface, but also other pollutants such as mud, spilled sand or hydrocarbon fuel or other operating fluids of the car, change the parameters of the surface roughness. Particularly dangerous are local changes in anti-slip properties of the surface caused by, for example, hydrocarbon fuel stains $[1,2,3]$. Special attention has been paid to it in the aspect of the safety of motorcyclists. In the United Kingdom, a social campaign was carried out on this topic [4], which also resulted in studies assessing whether the most commonly used pavement clean-up procedures satisfactorily restored the surface roughness $[2,4,5]$. The tests were carried out both in the laboratory using the English pendulum as well as in the real scale using two sliding cars and the SRT-3 device.

\footnotetext{
"Corresponding author: dgancarczyk@sgsp.edu.pl
} 
The report [4] pointed to the lack of procedures to assess whether the actions taken at the scene removed the slipperiness of the surface. It has been shown that the most frequently used methods of removing oil pollution from roads did not allow restoring the original anti-slip properties of the surface. The best effects were observed after the use of a mineral sorbent and complete collection of sorbent with absorbed liquid. The often used procedure, especially in large spills, consisting in leaving the saturated sorbent on the roadway increased the risk for road users. The inefficiency of using sand to remove the hydrocarbon fuel spill from the roadway has also been confirmed. Similar studies have also been carried out in other countries $[5,6,7]$.

In the majority of European countries, there are no regulations allowing to assess the effectiveness of the applied procedures of cleaning road surfaces in restoring the roughness of the surface. The exception is the French standard ANFOR NF P98-190: 2002 and the Norwegian standard DD CEN / TS 15366: 2009. These standards describe the procedures for testing the influence of sorbents on the friction coefficient of a surface. The English pendulum is used for research. In the case of the French standard, tests are carried out for the dry surface and pavement after removing the oil stain by means of a sorbent. It is permissible to reduce the original coefficient of friction by a maximum of $10 \%$ [8].

The Norwegian standard introduces two classes of sorbents depending on the degree of restoration of the coefficient of friction of the clean surface. The tests are carried out for three variants: clean surface, oily surface and sprinkled with sorbent, and for the surface after collecting sorbent with absorbed liquid. Class I allows a reduction in the coefficient of friction by a maximum of $10 \%$, while Class II allows a reduction in the coefficient of friction by $20 \%$ [9].

In the tests carried out at the SGSP, an attempt was made to determine the degree of friction coefficient restoration of polluted road surfaces using different sorption materials and the procedures for their use

\section{Materials and methods}

In order to investigate to what extent the applied sorbent restored the roughness of the surface, a series of coefficient friction measurements were carried out. For this purpose, a research station made in the Laboratory of Fire Extinguishing Agents was used, which is based on the inclined plane method. The research stand consisted of steel frame in which surfaces samples with dimensions of $350 \times 350 \times 50 \mathrm{~mm}$ were placed and the $9 \mathrm{~kg}$ steel rectangular sampler gummed with piece of a car tire. The frame was attached to the platform on one side by means of hinges, on the other hand, on a steel rod that allowed the angle of the plane to be changed [10]. The diagram of the test stand is shown in Figure 1. 


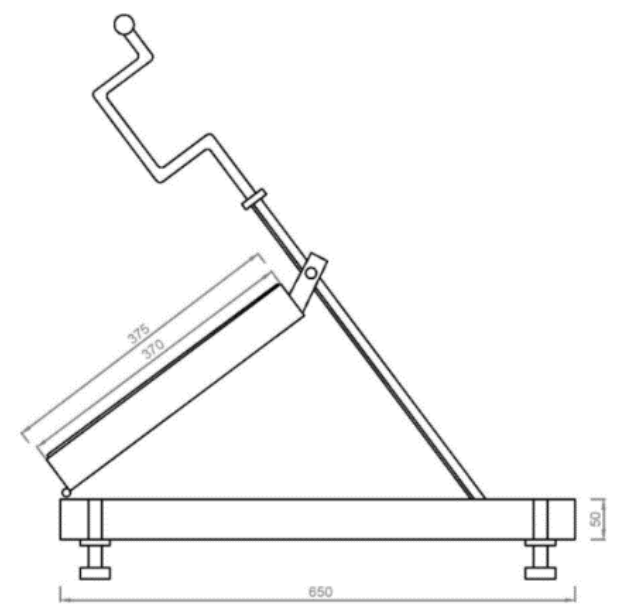

Fig. 1. Scheme of the test bench used to measure the coefficient of friction.

This position allows to easily determine the coefficient of static friction, which is equal to the tangent of the angle of inclination at the moment when the probe begins to slide. The angle was not measured directly, but was calculated based on the length of the side of the side. The length of the hypotenuse was constant and amounted to $375 \mathrm{~mm}$. The angle at which the probe began to slide was calculated from the relationship:

$$
\alpha=\operatorname{arcos}\left(\frac{a}{c}\right) * \frac{180}{\pi}\left[^{\circ}\right]
$$

where:

$\alpha$ - angle at which the probe slides off the ramp, a - side of the side marked in the tests, $\mathrm{c}$ - side of the hypotenuse with a length of $375 \mathrm{~mm}$.

Coefficient friction tests were performed for three different mineral-asphalt surfaces for dry, wet, oil-contaminated surfaces and for pavement after application of a sorbent. About $100 \mathrm{~cm}^{3}$ tap water was used to wet the samples. Water was spread evenly over the sample of the asphalt surface set in a horizontal position. Preparation of the oily surface test consisted of evenly spreading with a brush about $30 \mathrm{~cm}^{3}$ of vegetable oil used as a substitute for hydrocarbon fuel.. The thin layer causes a high slip imitating the slippery surface in the caused by the stain of diesel fuel on the roadway in real events and allows to observe the destructive effect of this type of substance on the tested asphalt surfaces. The last stage consisted of cleaning the oiled surface with a sorbent. For this purpose, the surface was first oiled as described above, then the oily surface was sprinkled with a sorbent and "mixed" with a brush. Sorbent, as in the case of real activities, was used in excess (about $60 \mathrm{~g}$ of professional sorbents, about $35 \mathrm{~g}$ of peat and sawdust and about $300 \mathrm{~g}$ of sand). After cleaning the surface, the oil-soaked sorbent was removed with a brush and the coefficient of friction was measured. Three professional sorbents and substitute materials were selected for the study. Table 1 presents the basic properties of the materials tested.

Table 1. The properties of the tested sorbents and substitute materials [11].

\begin{tabular}{|c|l|c|c|c|c|c|}
\hline No. & $\begin{array}{c}\text { Trade } \\
\text { name }\end{array}$ & Producer & $\begin{array}{c}\text { Sorption } \\
\text { capacity } \\
{[\mathbf{k g} / \mathbf{k g}]}\end{array}$ & $\begin{array}{c}\text { Granulation } \\
{[\mathbf{m m}]}\end{array}$ & $\begin{array}{c}\text { Bulk density } \\
{\left[\mathbf{k g} / \mathbf{d m}^{\mathbf{3}}\right]}\end{array}$ & $\begin{array}{c}\text { Humidity } \\
{[\mathbf{\%}]}\end{array}$ \\
\hline 1. & $\begin{array}{l}\text { Eco Dry } \\
\text { Compact }\end{array}$ & Reo Amos & 0.72 & $1-3$ & 0.576 & 0.416 \\
\hline 2. & Eco Dry & Reo Amos & 1.03 & $\sim 1$ & 0.491 & 0.257 \\
\hline
\end{tabular}




\begin{tabular}{|c|l|c|c|c|c|c|}
\hline & Plus & & & & & \\
\hline 3. & $\begin{array}{l}\text { Eco Dry } \\
\text { Aqua }\end{array}$ & Reo Amos & 1.18 & $0.3-0.7$ & 0.489 & 1.549 \\
\hline 4. & $\begin{array}{l}\text { Sawdust } \\
\text { pine }\end{array}$ & $\begin{array}{c}\text { Sawmill in } \\
\text { Przewrotne }\end{array}$ & 4.30 & $0.6-3.8$ & 0.138 & 9.868 \\
\hline 5. & Sand & Vistula sand & 0.18 & $\sim 1$ & 1.692 & 0.226 \\
\hline 6. & Peat & Oilex & 4.16 & - & 0.124 & 16.313 \\
\hline
\end{tabular}

${ }^{1}$ Podkarpackie voivodeship

Each measurement was started from lowering the incline and setting the gummed sampler, taking care to ensure that the sampler was in the same place on each trial. The next step was lifting the incline with a threaded rod until the probe started to slide. For this angle, the length of the side of the side with the accuracy of $1 \mathrm{~mm}$ was read. For each variant, 5 measurements of the coefficient of friction were made, and then the result was averaged.

Three samples of mineral-asphalt pavements were prepared for the tests, which were made in the form of cuboidal blocks with dimensions of 350x350x50mm:

- Surface of the QPR 2000 mineral-asphalt mix for cold repairs - the mixture was spread in layers about $2 \mathrm{~cm}$ thick and was manually beaten before applying the next layer. Then the sample was seasoned in a dry and well-ventilated place for 14 days.

- WMA mineral-asphalt surface (warm mix asphlat) - surface made in WMA heat technology . It was obtained during the replacement of the road surface in Dzikowiec in the Podkarpackie Voivodeship. After laying the asphalt in the form, it was compacted using a 7 ton roller. Then the sample was seasoned for two weeks.

- $\quad$ Surface used for about 30 years - surface made of SMA MATT / GRIS mix. The surface sample comes from the reconstructed Potocka street in Warsaw. It is a hightraffic road that has been used for around 30 years by both passenger cars and lorries. For the test, the sample was cut with a circular saw, cleaned of dust, washed and dried.

\section{Results and discussion}

The influence of applied sorbents on the restoration of surface roughness for the surface made of the QPR 2000 mixture is presented in Fig.2.

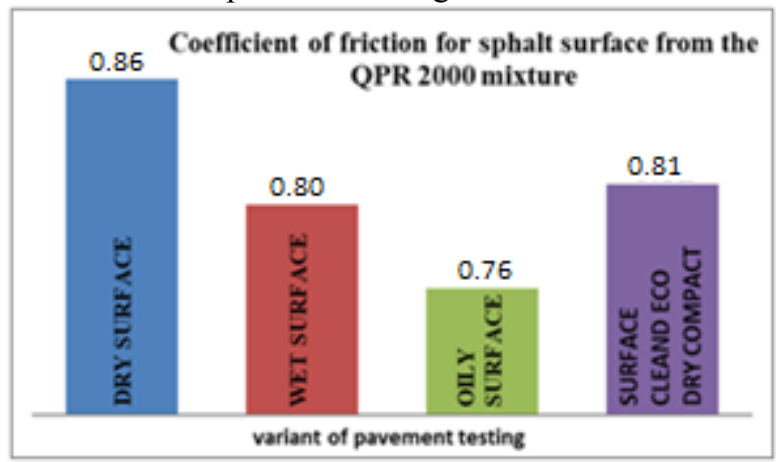

Fig. 2. A comparison of the effectiveness of sorbents used to restore the roughness of the asphalt surface from the QPR 2000 mixture.

This surface was the least resistant to the destructive effect of fuel on its structure. The oil washed out the binder and caused complete destruction of the prepared sample. Accordingly, no variants of the study were examined for this surface. 
Higher resistance to fuel was demonstrated by the WMA surface laid in the heat technology. This sample was also characterized by high porosity, which caused the penetration of both water and oil inside the structure. Also in this case, leaching of the binder was observed, but the process was much slower than in the QPR 2000 surface sample. The results of the friction coefficient for the WMA asphalt-mineral surface are shown in Figure 3.

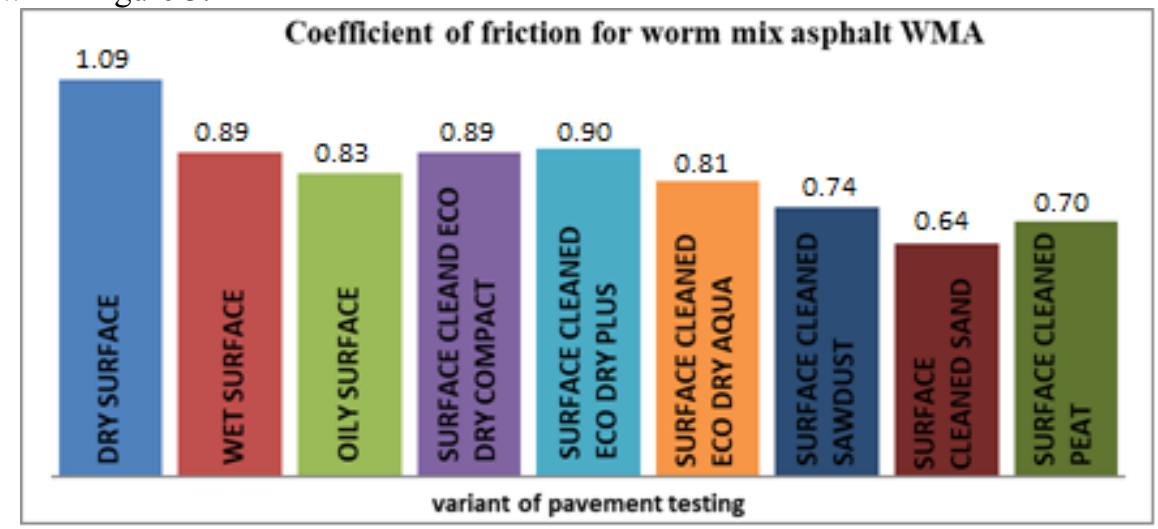

Fig. 3. A comparison of the effectiveness of sorbents used to restore the roughness of WMA asphalt mineral surface.

The highest effectiveness in removing slipperiness of the surface has been demonstrated by professional sorbents. These materials restored the coefficient of friction for the dry surface in about $80 \%$. Due to the porous structure of the tested surface, better surface restoration properties were observed for sorbents with higher granulation. ECO DRY AQUA sorbent, which was characterized by the smallest grain size, after absorption of oil, remained in the structure of the porous surface, reducing the coefficient of friction. Good effectiveness in restoring anti-slip properties, about $60 \%$ of the original coefficient of friction, was also observed after applying substitute materials - sawdust and peat. Due to the small size of the grains, these materials also remained in the structure of the porous surface, which prevented the complete cleanup of the contaminated sorbent. Similar conclusions were obtained in [6]. The most commonly used substitute material - sand, showed the weakest ability to restore the original coefficient of friction, the surface after using this material remained wet. The quantity that has been used to clean the surface (about 5 times greater than professional sorbents) is also significant.

The last test surface was a sample from a road with high traffic, used for about 30 years. The pavement sample was compacted and not characterized by a porous structure as previous surface samples. As a result, both oil and water did not seep through the sample and formed a slippery stain on the surface. This surface was characterized by the lowest coefficient of friction among the tested surfaces, whereas it showed the highest resistance to the destructive effect of fuel. A comparison of the effectiveness of sorbents used to restore the roughness of a surface used for a long period of time is shown in Figure 4. 


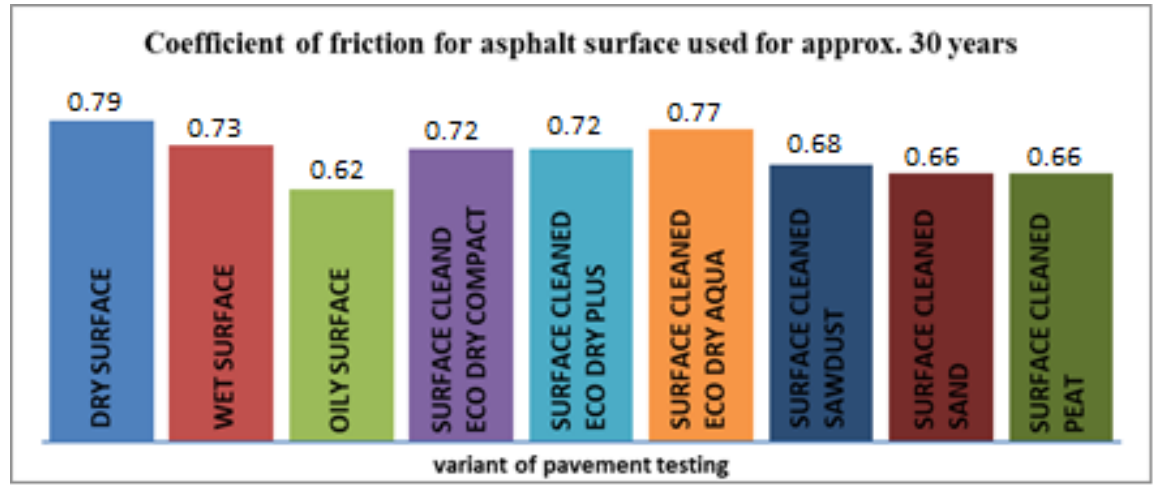

Fig. 4. A comparison of the effectiveness of sorbents used to restore the roughness of the asphalt surface, which was in continuous use for about 30 years.

As in the previous case, the best properties in removing slipperiness of the surface have been shown by mineral sorbents available on the market. Due to the compact structure of the surface, the highest efficiency was demonstrated by a sorbent with a low granulation Eco Dry Aqua, which allowed to restore the original coefficient of friction in $97 \%$. The use of substitute materials, i.e. sand and peat, slightly improved the anti-slip properties compared to the oil-coated surface.

The sorbents and substitute sorption materials used in the tests were characterized by different effectiveness in removing the slipperiness of the tested surfaces. When analyzing the obtained results of friction coefficient measurements, it should be noted that none of the used sorbents and substitute sorption materials has restored the value of the coefficient of friction determined for the dry surface. The best anti-slip properties were characterized by the newly placed WMA mineral-asphalt surface in the heat technology. Coefficient of friction of the surface used for about 30 years decreased by less than $30 \%$ in relation to the newly laid surface. Surfaces covered with oil were characterized by a coefficient of friction less by approx. $20 \%$ than dry surfaces.

\section{Conclusions}

The roughness of the surface is an extremely important parameter affecting road safety. The activities of fire protection units as well as other services when removing the spilled fluids from the roadway should therefore ensure the restoration of the original anti-slip properties of the surface. In Poland, there are no guidelines or applicable testing procedures, evaluating whether the sorbent used and the method of surface cleaning sufficiently removed the slipperiness of the surface. The authors point to the need to develop guidelines that would allow the assessment of surface roughness parameters after completed rescue operations. It is necessary to indicate the most reliable method and test bench testing the anti - slip properties of the surface.

The test stand used in tests allows to easily determine the coefficient of friction of the tested surface. The disadvantage of this method is the need to prepare pavement samples with specific dimensions. It is also not possible to assess the quality of cleaning up procedures of surface from spills in real conditions on roads. Therefore, it is necessary to continue research on the impact of the method of removing oil spills on the friction coefficient of the surface.

The tested surfaces were characterized by a different coefficient of friction. As expected, the best anti-slip properties were demonstrated by the new WMA mineral-asphalt surface. Long-term use of the road for about 30 years and high loads resulted in a 
significant reduction in the coefficient of friction. The new surfaces also showed little resistance to spilled fuel, which washed out the binder causing the top layer to crumble and damage the road. None of the used sorption materials did not restore the coefficient of friction of the dry surface. In addition, sorbents with low granulation after the absorption of dangerous liquid remained in the porous structure preventing the complete removal of the contaminated sorbent. Peat and sawdust showed significantly worse properties in restoring surface roughness than mineral sorbents, however, using them as ancillary materials when securing large spills or removing several kilometers of roadway oil may be justified

The worst property of restoring the coefficient of friction was demonstrated by sand. The road surface after the application of the sand remained wet. In addition to cleaning the surface, it was necessary to use several times more material, which increases the amount of contaminated material, which must be disposed of for disposal. Paradoxically, this increases the cost of the rescue operation.

\section{References}

1. Z. Ślosorz, K. Radwan, J. Rakowska, In: Problemy usuwania zanieczyszczeń ropopochodnych z infrastruktury drogowej i przemysłowej, 22 (2013)

2. J.C. Bullas, J. Doxford, P. Hupton, Project Report STAR 2014 (Highways Agency Manchester, 2014)

3. L. Seiler-Scherer, Conference paper Swiss Transport Research Conference 2004 (2004)

4. B. Meitei, M. Keigan, Project Report PPR509 (2010)

5. R.F. Lambourn, H.E. Viner, Project Report PPR073 (2006)

6. J. Kudrna, L. MALIŠ. In: Proceeding of conference. PIARC, 181 (2006)

7. D. Gancarczyk, M. Sobolewski, M. Słota. Zeszyty Naukowe SGSP 64, 7 (2017)

8. AFNOR NF P98-190:2002

9. DD CEN/TS 15366:2009

10. P. Książek, Badanie skuteczności sorbentów stosowanych do oczyszczania powierzchni utwardzonych, Engineering work (Warszawa 2018)

11. Reo Amos company material 